\title{
Design of UHF Fractal Antenna for Localized Near-Field RFID Application
}

\author{
Yonghui Tao, Erfu Yang, Yaxian Dong, and Gang Wang, Member, IEEE
}

\begin{abstract}
In this paper, fractal structure is proposed for localized near-field UHF antenna of radio frequency identification (RFID) reader. In the design, traditional Koch fractal structure is modified by converting the direction of one fractal structure of each line segment. Parameters of the fractal structure are optimized to acquire strong and uniform magnetic field distribution within an interrogation zone at designated height over the antenna. Two Koch fractal near-field antennas fed from center or side are presented. And the side-fed fractal near-field antenna is fabricated and tested to demonstrate a localized coverage area with a perimeter of two wavelengths within a reading distance of $35 \mathrm{~mm}$ above the antenna. For distance above $35 \mathrm{~mm}$, a sharp decrease of reading rate is observed, which demonstrates a satisfactory near-field UHF antenna.
\end{abstract}

Index Terms - Antenna, Koch Fractal, RFID, UHF

\section{INTRODUCTION}

$\mathrm{I}_{\mathrm{o}}^{\mathrm{N}}$ quite some of RFID-based applications, UHF RFID tags on objects must be identified in near field of a UHF RFID reader antenna [1,2]. For example, UHF RFID reader antenna on a level of smart shelf should restrict its coverage without interrogating tags of objects on other levels. With a localized coverage of near-field reader antenna, area on the level can be further partitioned with several localized RFID antennas so that exact object position on the shelf level can be acquired by interrogating logically the localized RFID antennas. Ordinary directional UHF antenna may not be suitable for such applications because it is generally designed for far-field operation.

In recent years, design of near-field UHF antennas for such RFID applications has received much attention, and several successful designs have been reported based on loop structure. Segmented loop with lumped capacitors [3], coupled lines [4], distributed capacitor [5,6], dash line [7,8], and a solid-line loop embedded phase shifter [9] are constructed to keep the current along the loop equal and in-phase to get strong and uniform

This work was supported in part by the National Natural Science Foundation of China under Grant 61272471

Yonghui Tao is with the Department of Engineering Science, University of Science and Technology of China, Hefei 230027, China.

Erfu Yang is with Space Mechatronic Systems Technology Laboratory (SMeSTech), Strathclyde Space Institute, Department of Design, Manufacture and Engineering Management, University of Strathclyde, 704 James Weir Building, 75 Montrose Street, Glasgow G1 1XJ, United Kingdom.

Yanxian Dong and Gang Wang are with Key Laboratory of Electromagnetic Space Information and Department of Electronic Engineering and Information Science, University of Science and Technology of China, Hefei 230027, China. (e-mail: gwang01@ustc.edu.cn). magnetic field over an electrically-large interrogation zone. However, it's hard to realize the same magnetic field intensity at the centre of the loop as the magnetic field in the regions close to the loop lines, which will cause a sharp gap. Meanwhile, it's shown that designing electrically-large near-field UHF RFID reader antenna is a big challenge, especially when the perimeter of interrogation zone reaches three wavelengths or more [10].

The majority of the ultrahigh frequency near-field RFID reader antenna designs are based on the theory that try to ensure the current along with the loop sharing same phase and direction by the means of adding various capacitors. For example, in 2007, Dobkin et al proposed a segmented loop antenna, with a diameter of $10 \mathrm{~cm}$ demonstrated desirable performance at $915 \mathrm{MHz}$, consists of a series of metal line sections and numbers of capacitors shaping a loop[3]. In[6], double-C shaped distributed capacitors. In 2013, Shi et al presented a dual loop antenna composed of a main and a parasitic loop which both are constructed using segmented lines with fork-shaped capacitors [11]. And then, they proposed a grid-array antenna composed of two segment loops to enlarge the interrogation zone [10].

The major challenge with design of a near-field UHF antenna is how to cover an electrically-large but still near-field region with strong and uniform magnetic field distribution. In this paper, we propose a near-field UHF RFID reader antenna based on Koch fractal structure. By optimizing the fractal structure, reading nulls should be avoided in the entire covered near-field region. Outside the restricted near-field region, the field strength or the read area should decrease as fast as possible so that a more precise position of the RFID tag can be located.

\section{KOCH FRACTAL CONFIGURATION}

Basic Koch fractal structure is depicted in Fig. 1(a). For the need of designing near-field UHF RFID reader antenna, we modified the Koch fractal structure properly. As depicted in Fig. 1(b), the modified Koch fractal can be defined by four variable namely $b(\mathrm{~mm}), \mathrm{a}(\mathrm{mm}), N($ recursive total number) and $\alpha$. And the direction of one fractal structure of each line segment is changed, which can make the Koch curve more flexible and homogeneous to fill the space. At the same time, to lessen the chance of structure overlapping in the antenna optimization process, as the recursive total number $(N)$ increases each time, only the lines generated in last fractal process will be replaced by fractal curves.

The advantages of using Koch fractal structure for UHF RFID reader antenna lie in its self-similarity and filling-ability. 
Due to the self-similarity, the four parameters can build a model.

When the Koch fractal is used as a UHF antenna due to the travelling-wave current on the fractal, radiation will occur at each turn of fractal structure. Therefore, by properly designing the fractal geometry, uniform near-field coverage can be acquired over the area covered by the Koch fractal.

As an antenna, Koch fractal can be fed either from the center as shown in Fig. 2(a), or from the side as shown in Fig. 2(b). For the side-fed structure it is quite convenient to enlarge the near-field coverage area by forming networks.

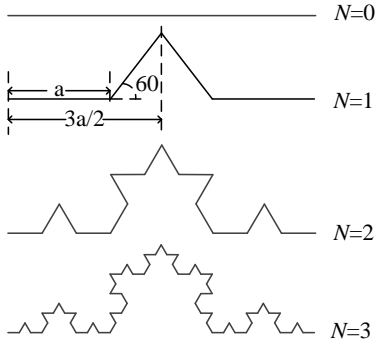

(a)

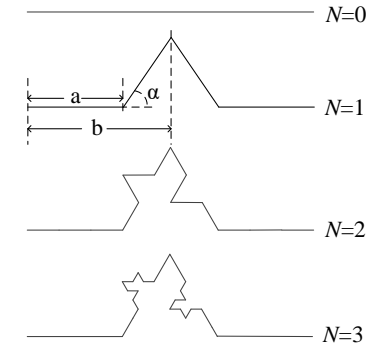

(b)
Fig. 1 (a) Basic Koch fractal structure,(b) modified Koch fractal structure .

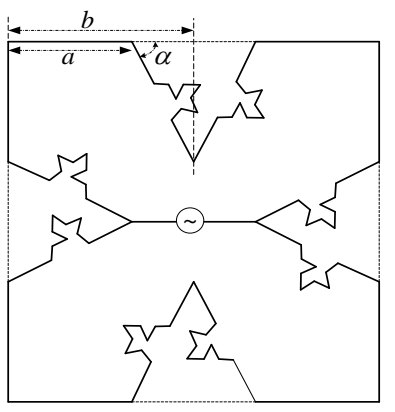

(a)

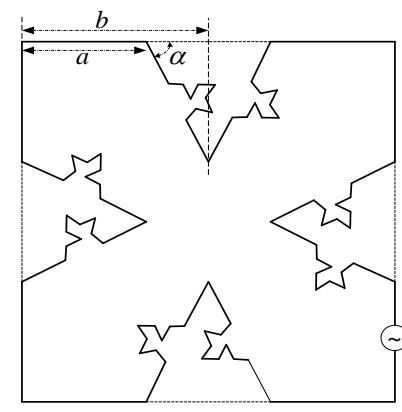

(b)
Fig. 2 Koch fractal antenna fed from the center (a), or from the side(b) .

\section{Koch Fractal NEAR-FIELD ANTENNA DESIGN}

Filling ability is another feather that help facilitating the structure uniformity of the interrogation zone. In this study, to acquire a square interrogation zone, the modified Koch fractal transformation is applied to every side of a square with a perimeter of approximately $2 \lambda$ at $915 \mathrm{MHz}$ (viz., the size of the antenna is $160 \mathrm{~mm} \times 160 \mathrm{~mm}$ ).

The combination of model code and 4NEC2's source code contributes to the rapid optimization and good results [12]. In this simulation, only one parameter is kept unchanged, while three parameters are varied at a time. In the code, ranges of the three parameters have been set. When the process is on, the figures of these ranges could be optimized. It is illustrated that the good results will be found as long as the boundary conditions problems being settled.

The objective functions for impedance matching $\left(f_{1}\right)$ and magnetic field distribution $\left(f_{2}\right)$ are defined as

$$
\begin{gathered}
f_{1}=\frac{10}{\left|S_{11}(d B)\right|} \\
f_{2}=\max \left(\frac{\min _{\Omega}\left|H_{z}(d B)\right|}{60}, \frac{\max _{\Omega}\left|H_{z}(d B)\right|-\min _{\Omega}\left|H_{z}(d B)\right|}{2}\right)
\end{gathered}
$$

where $S_{11}$ means return loss at $915 \mathrm{MHz}, H_{z}$ represents $z$-component of magnetic field to be designed for uniform coverage, $\Omega$ represents the area to be covered in near-field region.

\section{A. Center-fed Koch-Fractal Antenna}

Firstly, we consider a center-fed Koch-Fractal Antenna covering a near-field square. The model of the antenna is shown in Fig. 2(a).

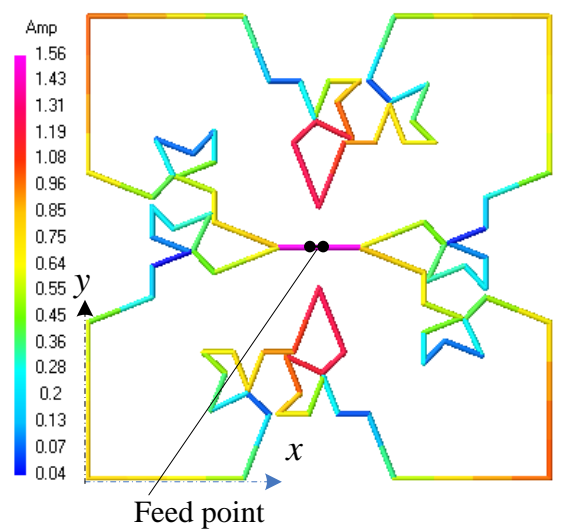

Fig. 3 Optimized center-fed Koch fractal antenna structure and its current distribution.

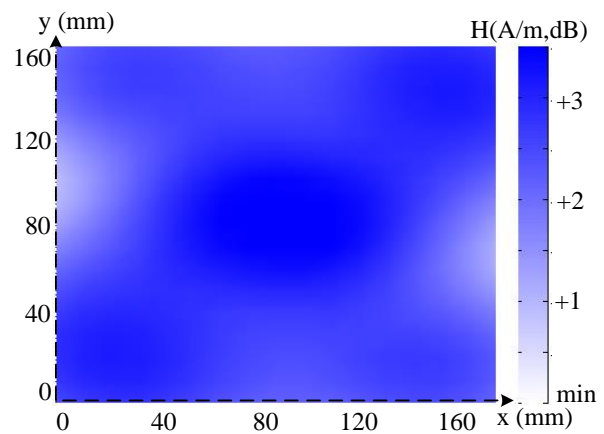

Fig. 4 Dynamic range of distribution of magnetic field intensity on plane $z=$ $30 \mathrm{~mm}$.

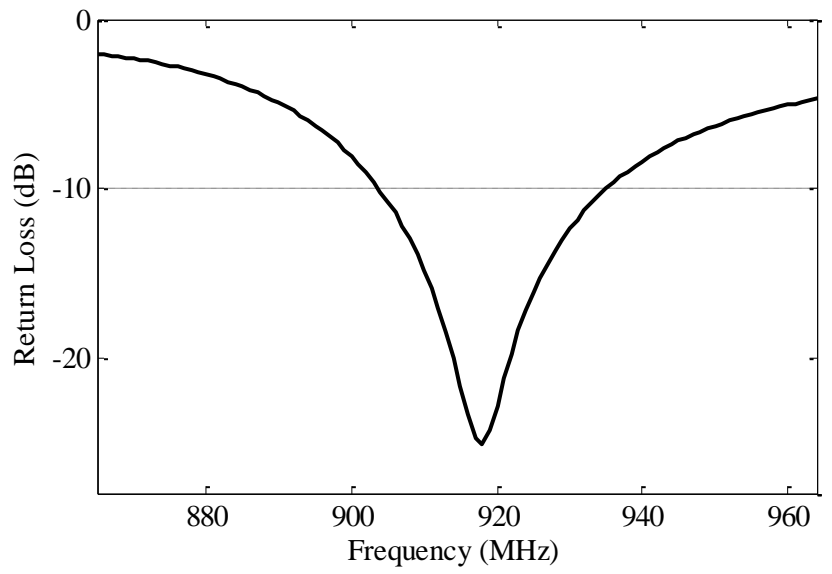

Fig. 5 Return loss of the center-fed Koch fractal antenna.

Fig. 3 shows the layout of optimised center-fed Koch-fractal antenna $(\alpha=68.45, a=53.6 \mathrm{~mm}, b=80 \mathrm{~mm}$, ratio $=a / b=0.67)$ and the current distribution. Fig. 4 shows the distribution of amplitude of z-component of magnetic field, $|\mathrm{Hz}|(\mathrm{A} / \mathrm{m})$, in $\mathrm{dB}$ on the $x y$-plane of $z=30 \mathrm{~mm}$. And the dynamic range of $|\mathrm{Hz}|$ is measured to be less than $3.5 \mathrm{~dB}$ in the whole interrogation 
zone $(0 \leq x \leq 160 \mathrm{~mm}, 0 \leq y \leq 160 \mathrm{~mm})$. Dynamic range of $3.5 \mathrm{~dB}$ indicates a quite satisfactory uniform coverage, which is superior to the results acquired in the present UHF near-field RFID reader antennas.

Fig. 5 shows the simulated return loss at $915 \mathrm{MHz}$. It is observed that the optimised center-fed Koch-fractal antenna has a satisfactory input impedance matching over frequency band 904-930 MHz.

\section{B. Side-fed Koch-Fractal Antenna}

However, in practical application, multiple RFID reader antennas may work together to enlarge the interrogation zone or realize hierarchical and regional position. In these cases, side-feeding should be adopted to facilitate placing and feeding antennas. Therefore, we consider a side-fed Koch-fractal antenna as shown in Fig. 2(b).

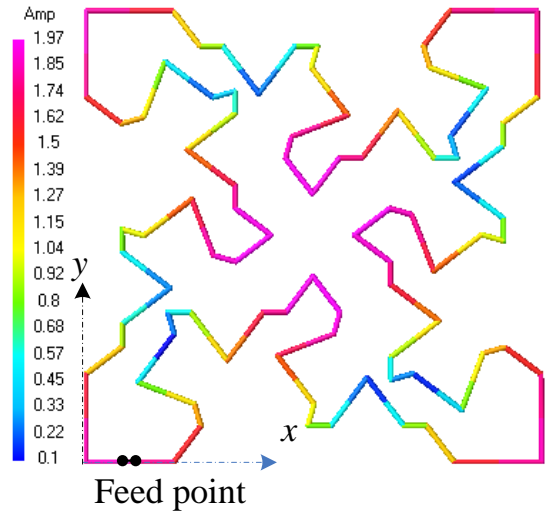

Fig. 6 Optimized side-fed Koch fractal antenna structure and its current distribution.

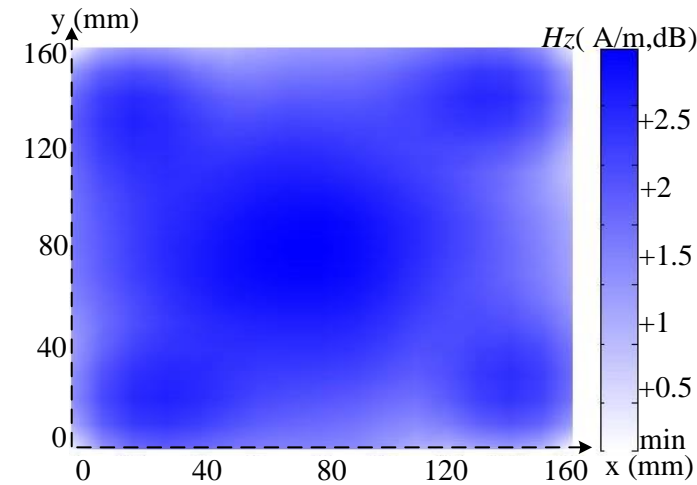

Fig. 7 Dynamic range of distribution of magnetic field intensity on plane $\mathrm{z}=$ $30 \mathrm{~mm}$.

Fig. 6 shows the layout of optimised side-fed Koch-fractal antenna $(\alpha=54.28, a=32.8 \mathrm{~mm}, b=80 \mathrm{~mm}$, ratio $=a / b=0.41)$ and the current distribution. The Fig. 7 shows the distribution of amplitude of z-component of magnetic field, $|\mathrm{Hz}|(\mathrm{A} / \mathrm{m})$, in $\mathrm{dB}$ on the xy-plane of $z=30 \mathrm{~mm}$. From Fig. 7, it is measured that the dynamic range of $|\mathrm{Hz}|$ is within $3 \mathrm{~dB}$ in the whole interrogation zone $(0 \leq x \leq 160 \mathrm{~mm}, 0 \leq y \leq 160 \mathrm{~mm})$.

Fig. 8 illustrates the simulated return loss at $915 \mathrm{MHz}$. It is found that the optimised Koch-fractal antenna has a satisfactory input impedance matching over frequency band 909-920 MHz.

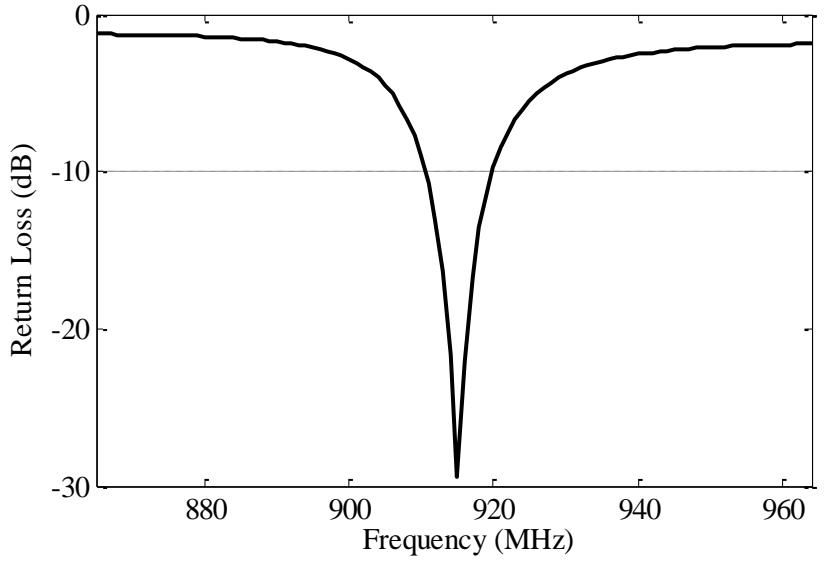

Fig. 8 Return loss of the side-fed Koch fractal antenna.

In conclusion, by applying modified Koch-fractal structures, the UHF RFID antenna can provide perfect impedance matching, as generating magnetic field distribution being more uniform than that of traditional near-field UHF loop antennas [8-12]. That's because the filling ability of modified Koch fractal structure can help antenna improve the structure uniformity. And owing to the Koch fractal structure's self-similarity, a model of the antenna is built to optimize Koch fractal structures to acquire optimized current distribution, which can generate magnetic field in interrogation zone as uniform as possible.

\section{FABRICATION AND TEST}

Prototype of the designed side-fed Koch-fractal antenna is fabricated by using copper wires on a paper board, as shown in Fig. 9. The paper board has dimensions of $190 \times 190 \mathrm{~mm}$, thickness of $3.5 \mathrm{~mm}$, and dielectric constant of $\varepsilon_{r}=2.5$. At the feeding port, an SMA connector of $50 \Omega$ input impedance is directly connected to the two ends of the feeding copper wire of the antenna, as shown in the zoomed picture in Fig. 9.

The impedance matching of the prototype was measured using Agilent E5230A vector network analyzer. The simulated and measured return loss of the antenna plotted in Fig. 10, show good agreement.

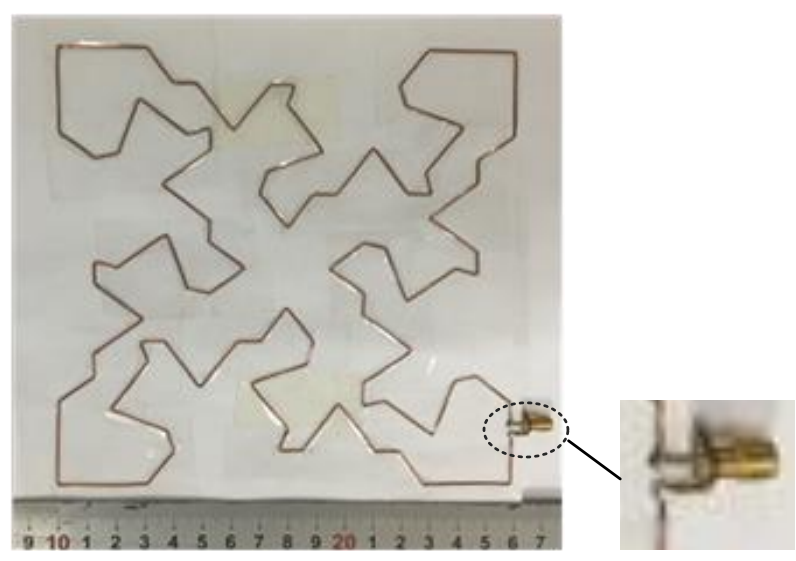

Fig.9 Photo of the side-fed Koch-fractal antenna prototype. 


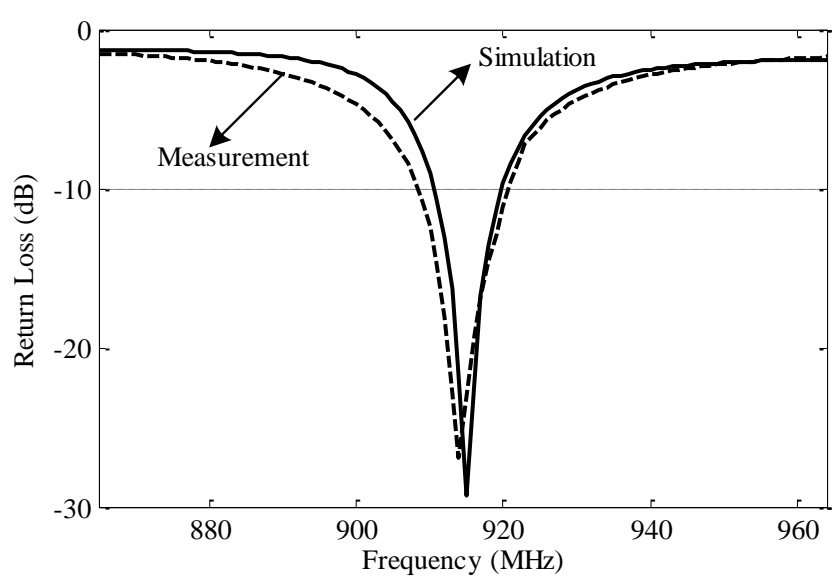

Fig. 10 Simulated and measured $\left|\mathrm{S}_{11}\right|$ of the side-fed Koch-fractal antenna.

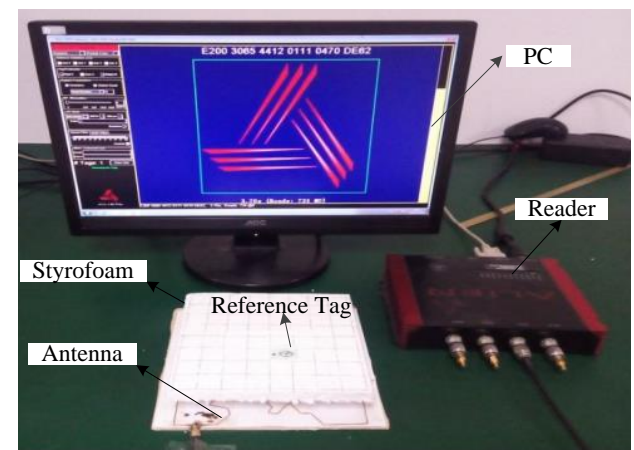

(a)

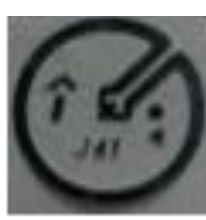

(b)
Fig. 11 Near-field RFID measurement setup (a) and reference tag (b).

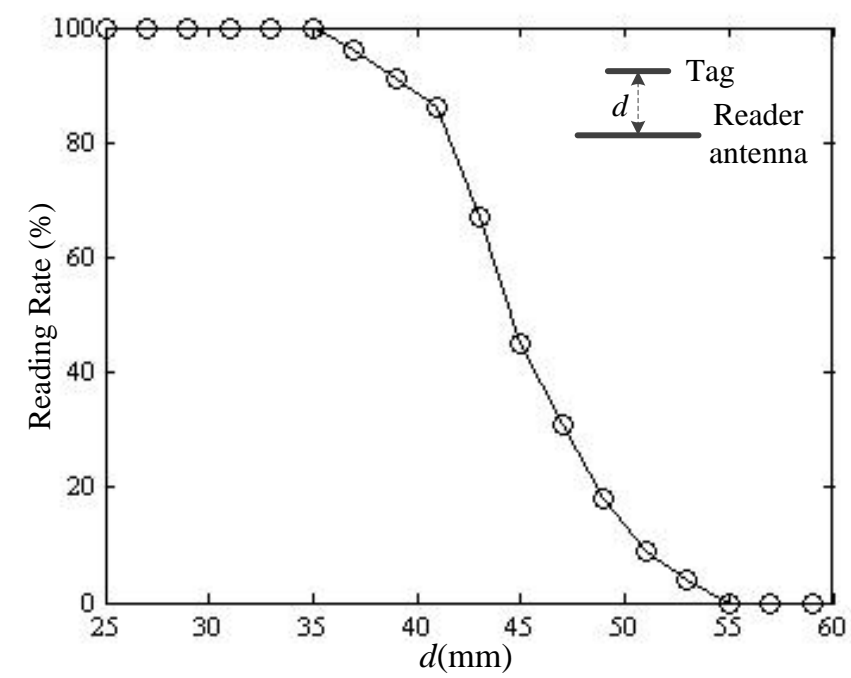

Fig. 12 Measured reading rate against reading distance $(d)$ of the side-fed Koch-fractal antenna prototype.

To verify the near-field coverage performance, the prototype of designed side-fed Koch-fractal antenna is applied to detect a single reference tag. In the detection setup shown in Fig. 11, the antenna prototype is connected to Alien ALR 9900+ RFID reader, which may operate over 902-928 MHz RFID band with an output power of $24 \mathrm{dBm}$. Impinj button type near-field UHF tag, J41, with a loop-like antenna of diameter of $12 \mathrm{~mm}$, is taken as reference tag.

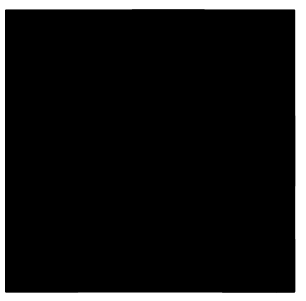

(a)

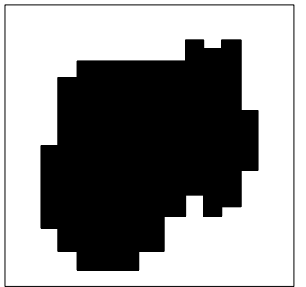

(c)

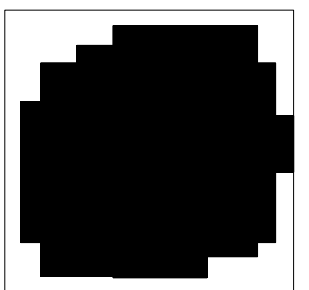

(b)

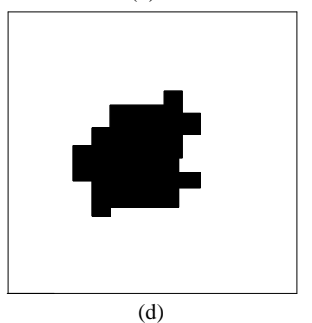

Fig. 13 Distribution of active grids on styrofoam plate at several typical reading distance d (a) $d=35 \mathrm{~mm}$, (b) $d=40 \mathrm{~mm}$, (c) $d=45 \mathrm{~mm}$, (d) $d=50 \mathrm{~mm}$.

As shown in Fig. 11(a), a single J41 tag is supported by a square Styrofoam plate with dimension of $160 \times 160 \times 15 \mathrm{~mm}$ and $\varepsilon_{r}=1.11$. On the Styrofoam plate, local coordinates is set and the Styrofoam plate is gridded for easy positioning of the tag on the xy-plane. By raising the plate to different height, and deploying the reference tag at different coordinates in turn on the square Styrofoam, face-to-face to the Koch-fractal reader antenna, the coordinates(active grids) at which the reference tag can be interrogated by the RFID reader can be measured. And then by further analyzing the distribution of active grids, the near-field coverage performance of antenna prototype can be evaluated.

Fig. 12 depicts the measured reading rate against reading distance $(d)$ of the side-fed Koch-fractal antenna prototype. It is observed that a $100 \%$ reading rate is achieved within a maximum distance of $35 \mathrm{~mm}$. Furthermore, as the reading distance is above $35 \mathrm{~mm}$, the reading rate rapidly declines from $100 \%$ of $35 \mathrm{~mm}$ to $0 \%$ of $55 \mathrm{~mm}$. Such a sharp decrease is beneficial for tag orientation and could be hard to obtain for conventional Near-field UHF loop antennas [8-12].

The distribution of the active grids marked by black squares of dimensions of $10 \times 10 \mathrm{~mm}$ at several typical reading distance ((a) $d=35 \mathrm{~mm}$, (b) $d=40 \mathrm{~mm}$, (c) $d=45 \mathrm{~mm},(\mathrm{~d}) d=50 \mathrm{~mm}$ ) is shown is Fig. 13. It is observed that there is no reading null at the reading distance of $35 \mathrm{~mm}$. Furthermore, it is also found that there is no reading null in the middle of the Styrofoam plate at different reading distance. This is coincided with the simulation results depicted in Fig. 7, that with the designed side-fed Koch-fractal antenna larger magnetic field intensity can be acquired in the region around the centre of the interrogation zone, rather than around the outside frame.

\section{CONCLUSION}

The major challenge with design of a near-field UHF antenna is how to cover an electrically-large but still near-field region with strong and uniform magnetic field distribution. Fractal structures create a lot of possibilities for the antenna designs. In this paper, design of near-field UHF RFID antennas based on modified Koch fractal structure is presented. The simulation and experiment results indicate that the optimised Koch-fractal antennas fed from the center or side both can generate a 
localized coverage area with dimension of $0.5 \times 0.5 \lambda$ within a reading distance of $35 \mathrm{~cm}$ above the antenna. As providing perfect impedance matching, they generate magnetic field distribution being more uniform than that generated by conventional loop antennas.

Koch fractal structure is very promising for near-field UHF RFID applications. Firstly, the filling ability of fractal structures can help facilitating the structure uniformity of the interrogation zone. And then due to the self-similarity, fractal structures' parameters can be used to build a model, which combined with 4NEC2's source code to obtain excellent results rapidly.

\section{ACKNOWLEDGMENT}

This work was supported in part by the National Natural Science Foundation of China under Grant 61272471.

\section{REFERENCES}

1. P. V. Nikitin, K. V. S. Rao, and S. Lazar, "An overview of near field UHF RFID," in Proc. IEEE Int. Conf. RFID, pp. 167-174, 2007.

2. X. Qing and Z. N. Chen, "UHF near-field RFID antennas," in Proc. Int. Workshop Antenna Technol., pp. 1-4, 2010.

3. M. Dobkin, S. M. Weigand, and N. Iyec, "Segmented magnetic antennas for near-field UHF RFID,” Microw. J., vol. 50, no. 6, 2007.
4. R. A. Oliver, "Broken-loop RFID reader antenna for near field and far field UHF RFID tags," U.S. design patent D570, 337 S, Jun, 2008.

5. Y. S. Ong, X. Qing, C. K. Goh, Z. N. Chen, "A segmented loop antenna for UHF near-field RFID," in Proc. IEEE Antennas Propag. Soc. Int. Symp., pp. $1-4,2010$.

6. X. Qing, Z. N. Chen, and C. K. Goh, "UHF near-field RFID reader antenna with capacitive couplers," IET Electron. Lett., vol. 46, no. 24, pp. 1591-1592, Dec. 2010.

7. X. Qing, C. K. Goh, and Z. N. Chen, "Segmented loop antenna for UHF near-field RFID applications," IET Electron. Lett., vol. 45, no. 17, pp. 872-873, 2009.

8. X. Qing, C. K. Goh, and Z. N. Chen, "A broadband near-field UHF RFID antenna," IEEE Trans. Antennas Propag., vol. 58, no. 12, pp. 3829-3838,2010.

9. Z. N. Chen, C. K. Goh, and X. Qing, "Loop antenna for UHF near-field RFID reader," in Proc. 4th Eur. Conf. Antenna Propag., Barcelona, Spain, pp. 1-4, 2010.

10. J. Shi, X. Qing, and Z. N. Chen, "Electrically large zero-phase-shift line grid-array UHF near-field RFID reader antenna," IEEE Trans. Antennas Propag., vol. 62, no. 4, pp. 2201-2208, 2014.

11. J. Shi, X. Qing, Z. N. Chen, and C. K. Goh, "Electrically large dual-loop antenna for UHF near-field RFID reader," IEEE Trans. Antennas Propag., vol. 61, no. 3, pp. 1019-1025, 2013.

12. D. Ding, Y. H. Tao, G. Wang, "Design of near-field ultra-high frequency radio-frequency identification antenna with fragmented wires for electrically-large coverage," IET Microw. Antennas Propag., vol. 10, pp. 1218-1224, 2016. 